\title{
Gestión municipal y planificación urbana en Córdoba, Argentina (1983-2011)
}

\author{
Gestão municipal e planificação urbana em Córdoba, Argentina (1983-2011) \\ Urban management and urban planning in Córdoba, Argentina (1983-2011)
}

Martín Lemma ${ }^{[0, b]}$

[a] Consejo Nacional de Investigaciones Científicas y Técnicas (CONICET), Córdoba, Argentina

[b] Universidad Nacional de Córdoba (UNC), Instituto de Investigación de la Vivienda y el Hábitat (INVIHAB), Córdoba,

Argentina

\section{Resumen}

En 1983, Argentina retorna a la democracia y, a partir de entonces, diversos partidos políticos cordobeses estuvieron a cargo de la gestión de la Municipalidad de la Ciudad de Córdoba. Cada uno de ellos ha abordado, de una manera particular, los diversos problemas del espacio urbano del municipio. A lo largo de 28 años, estos abordajes se han ido modificando en mayor o menor medida, ya sea a modo de continuidad, transformación o ruptura. Hasta 1999, hubo en Córdoba una sucesión de gobiernos de un mismo partido político que se tradujo, en gran medida, en la continuidad de las políticas urbanas. Sin embargo, desde entonces y hasta 2011, se corta dicha sucesión de partidos y las tres gestiones municipales siguientes presentaron sus propias miradas urbanas y, en consonancia con ellas, intentaron transformar los marcos regulatorios. Este artículo busca analizar las diferentes maneras de pensar la forma urbana, intentando comprender los modelos físico-espaciales de la ciudad deseada por cada gestión municipal ante la constante problemática de crecimiento urbano por expansión de baja densidad habitacional que, de manera descontrolada, caracterizó la ciudad de Córdoba. Con este fin, se describen, se presentan de manera gráfica y se analizan los diversos documentos de planificación urbana publicados durante casi tres décadas, haciendo foco en las particularidades de cada documento y estableciendo vínculos con otros documentos del mismo periodo de tiempo.

Palabras clave: Planificación urbana. Morfología urbana. Córdoba.

\section{Resumo}

Em 1983, a Argentina voltou à democracia e, a partir desse momento, vários partidos políticos cordobenses foram responsáveis pela gestão do município de Córdoba. Cada um deles abordou os problemas do espaço urbano do município de uma forma particular. Em 28 anos, estas abordagens têm sido modificadas em maior ou menor grau, por meio de continuidade, transformação ou ruptura. Até 1999, houve em Córdoba uma sucessão de governos do mesmo partido político, que resultou em grande parte na continuidade das políticas urbanas. No entanto, desde então e até 2011, corta-se essa sucessão de partidos e as três administrações municipais seguintes apresentaram os seus próprios olhares urbanos e tentaram transformar os marcos regulatórios de acordo com eles. Este artigo procura analisar as diferentes formas de pensar a forma urbana, buscando

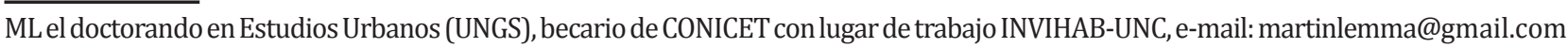


compreender os modelos físico-espaciais da cidade desejada por cada gestão municipal para enfrentar o problema constante de crescimento urbano por meio da expansão de baixa densidade habitacional de forma descontrolada que caracterizou a capital provincial. Para este efeito, são descritos, apresentados graficamente e analisados os documentos de planejamento urbano publicados durante estas quase três décadas. O foco é feito nas particularidades de cada documento e são estabelecidos pontos comuns com outros documentos do mesmo corte do tempo.

Palavras-chave: Planejamento urbano. Morfologia urbana. Córdoba.

\section{Abstract}

In 1983, Argentina returned to democracy and, thereafter, different political parties were responsible for the management of the Municipalidad de Cordoba. Each of them has approached the various urban spatial problems of the municipality in a particular way. Over 28 years, these approaches have been modified to a greater or lesser extent, either through continuity, transformation or rupture. Until 1999, there was a succession of governments from the same political party in Cordoba which resulted largely in continuity of urban policy. However, from then on until 2011, this succession process ended and the following three municipal administrations presented their own urban visions and, from these, attempted to transform the urban regulatory framework. This article seeks to analyze the different ways of thinking about urban form, seeking to understand the physical and spatial models of the city desired by each municipal management to face the constant problem of urban growth due to using low housing density in an uncontrolled manner. For this purpose, various urban planning documents published during these three decades are described, presented graphically and analyzed. These lectures focus on the particularities of each document and establish links with other documents from the same period.

Keywords: Urban planning. Urban morphology. Cordoba.

\section{Introducción}

Con el retorno de Argentina a la democracia en 1983, las diversas gestiones municipales de Córdoba han tenido que afrontar el problema de la incorporación de nuevos espacios urbanos para la ciudad en respuesta a las demandas de los cordobeses. El Municipio de Córdoba se encuentra situado sobre una llanura, al límite de una cadena montañosa denominada Sierras Chicas y cuenta con una población que, durante el período 1983-2011, creció de aproximadamente de los 0,99 a los 1,32 millones de personas (INDEC, 2010). Este gran crecimiento en términos absolutos, sin embargo, representa el comienzo de una caída de la tasa de crecimiento poblacional.

Cada gobierno municipal ha definido su propia manera de planificar la ciudad, ya sea a modo de continuidad, transformación o cambio con su propia gestión o gestiones anteriores. Este artículo se propone discutir las ideas de los productos tangibles de estas definiciones. A su vez, entiende que las mismas son también el resultado de un contexto político, social y económico, y de diferentes procesos de reflexión que las anteceden y cuya continuidad - o incluso ruptura - a menudo pudo ser garantizada por la continuidad ciertos equipos técnicos dentro de las áreas de planificación urbana municipal.

El esquema clásico de planificación urbana cuenta con instancias de diagnóstico, de diseño urbano, de regulación y de aplicación. Estas instancias no siempre se traducen de manera coherente a lo largo de este proceso, generando tensiones - y hasta contradicciones dentro del proceso de la planificación urbana. A lo largo de este artículo se describirán las diferentes maneras de pensar forma urbana para la Municipalidad de Córdoba durante el período 1983-2011, buscando comprender los modelos físico-espaciales de ciudad deseada por cada gestión municipal.

El modelo de crecimiento físico que caracteriza a la mancha urbana de Córdoba responde a un modelo teórico que fue presentado por primera vez en el documento Diagnóstico tentativo y alternativas de desarrollo 

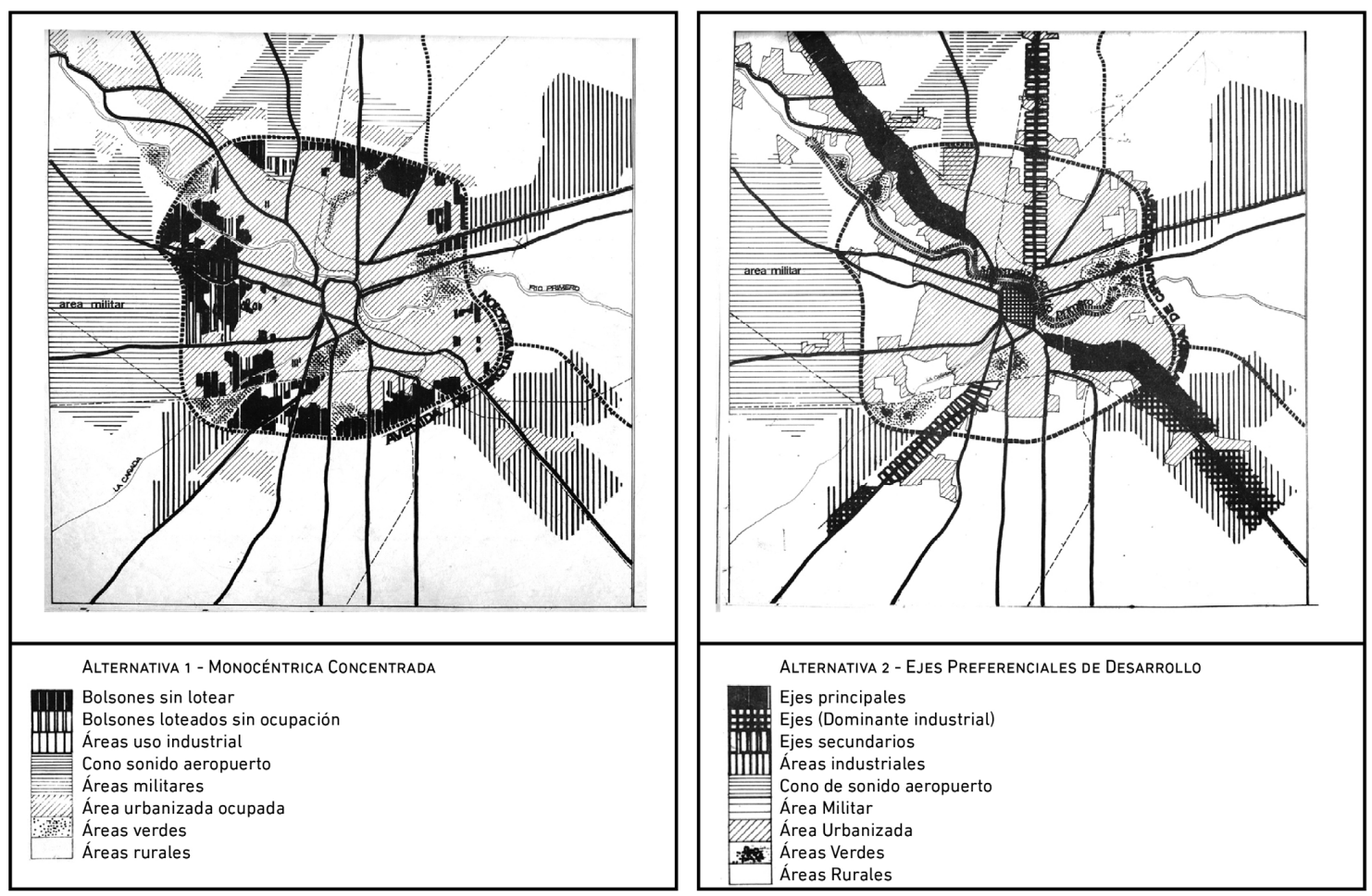

Figura 1 - Alternativa 1: Modelo radio céntrico concentrado - Alternativa 2: Ejes preferenciales de desarrollo Fuente: Elaboración propia a partir de gráficos de Diagnóstico tentativo y alternativas de desarrollo físico para la ciudad de Córdoba, Municipalidad de Córdoba (1973).

físico para la ciudad de Córdoba (Córdoba, 1973). En este documento se proponen dos alternativas formales: "Modelo radio céntrico concentrado" y "Ejes preferenciales de desarrollo" (Figura 1). La segunda de ellas será la que toma mayor fuerza dentro de las futuras instancias de planificación municipal; incluso ésta idea es la que se aprueba como Esquema de Ordenamiento Urbano para la ciudad de Córdoba: año 2000 (EDOU) (Córdoba, 1978) mediante una ordenanza municipal en 1978. La noción de los ejes preferenciales de desarrollo del EDOU se justifica por la lectura que se hace de la ciudad de Córdoba como centro metropolitano; esta idea aparece fuertemente en el Esquema Director de Ordenamiento Metropolitano (EDOM) (Córdoba, 1979) ${ }^{1}$.

\footnotetext{
${ }^{1}$ Antes del EDOU, existieron: Córdoba. Plan Regulador y de Extensión - formulado por la oficina técnica del Ing. Benito Carrasco en 1927 - y el Plan Regulador de la Ciudad de Córdoba - a cargo del Arq. Ernesto La Padula entre 1954 y 1962 -. Venturini et al. (2010) analiza estos y otros documentos de planificación urbana desde un enfoque de la sustentabilidad en el desarrollo de la ciudad; Díaz Terreno (2011) lo hace con
}

En el EDOU se piensa que los ejes de desarrollo serán los que trabajen como conectores entre la ciudad central y sus satélites. De esta forma, estos ejes radiocéntricos estructurarán la mancha urbana de la ciudad, cargándose de funciones urbano-metropolitanas y alojando el crecimiento habitacional mediante el incremento de la densidad residencial a sus márgenes. Posteriormente, se adjudicarán patrones morfológicos edificatorios a las áreas resultantes de esta división de la mancha. Los patrones responden a una lógica de ordenación de un centro con altas densidades habitacionales y una periferia de bajas, siempre atravesada por los corredores. Esta zonificación tenía también como objetivo frenar la urbanización de áreas periféricas libres en la medida en que se completaban las vacancias internas. En líneas generales, este modelo de crecimiento - junto con los marcos

una mirada histórica de cómo se conformaron los territorios periurbanos de la ciudad; Caporossi (2008) incorpora una mirada crítica sobre la planificación y el crecimiento urbano. En los tres casos, el recorte temporal comienza con el plan de Carrasco y finaliza con el cambio de siglo. 


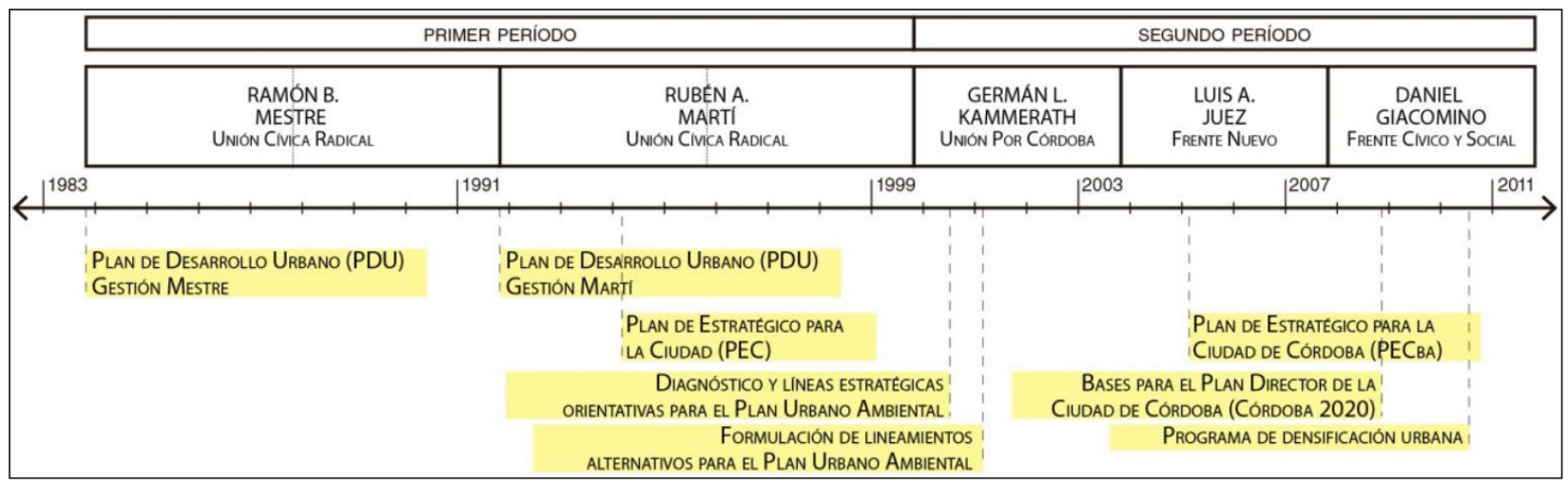

Figura 2 - Línea del tiempo con documentos de planificación urbana presentados en cada gestión municipal de Córdoba (1983-2011) Fuente: Elaboración propia.

regulatorios que resultan de él - tienen fuertes bases en modelos de planificación tradicional. Es decir, parten de la búsqueda de un modelo físico-espacial cerrado y utilizan la zonificación como herramienta de organización de formas y funciones.

En este artículo se revisan los documentos de planificación urbana publicados entre 1983 y 2011, buscando entender cuál es el modelo físico-espacial implícito en ellos y en su manera de proyectar la ciudad. A partir de la periodización que marcan las gestiones de la Municipalidad de Córdoba es posible comprender las diferentes maneras de pensar su forma urbana. En este sentido y como indica la Figura 2, hay un primer período que transcurre desde 1983 a 1999 en el cual tiene fuerza principalmente la idea de los ejes preferenciales de desarrollo, y un segundo período, 1999-2011, cuando diferentes ideas se solapan más claramente entre sí ${ }^{2}$. En términos de lo que sucede con la política urbana, la principal diferencia entre ambos períodos es el grado de continuidad de los partidos políticos: mientras que en el primer período se suceden cuatro gestiones de un mismo partido político, en el segundo período, el municipio fue gestionado por tres fuerzas políticas diferentes. Estas continuidades y discontinuidades del plano político tienen un reflejo casi directo en lo que ocurre en las maneras de pensar la forma urbana y es por ello que resultan útiles como recortes temporales para estudiar la evolución de los documentos de planificación urbana de Córdoba. Cabe resaltar que,

\footnotetext{
${ }^{2}$ En el Municipio de Córdoba, a partir del retorno a la democracia en 1983 y hasta la fecha, las gestiones municipales fueron de cuatro años y su fecha de cambio de mandato fue siempre los días 10 de diciembre.
}

a la par que se suceden estas transformaciones en el plano político, existe cierta continuidad de los equipos técnicos de las áreas de planificación urbana municipal cuyo accionar ha trascendido transversalmente las mutaciones políticas, colaborando en el flujo de ideas que se transfieren a los documentos de planificación presentados y que se analizan en este artículo.

\section{Primer período: una sucesión de gestiones del radicalismo}

Las cuatro gestiones que forman parte del primer período son, primero, dos gestiones de Ramón B. Mestre y, luego, dos de Rubén A. Martí, ambos pertenecientes a la Unión Cívica Radical. La gestión municipal de Ramón B. Mestre dio inicio a sus políticas urbanas con el denominado Plan de Desarrollo Urbano (PDU), de 1993. De este plan no se han podido encontrar documentos oficiales previos a su implementación, pero sí existe una publicación posterior de Guillermo Irós (1991), quien fue el Secretario de Desarrollo Urbano de la Municipalidad durante estas dos gestiones. La primera medida importante que se realizó dentro de este plan fue frenar la aprobación de nuevas urbanizaciones - salvo aquellas que fuesen de planes masivos de vivienda - provisoriamente por seis meses. Durante estos meses, se retomaron las instancias de diagnóstico y planificación que habían producido el EDOU, y se conformó un marco normativo de regulación del espacio urbano y de sus usos bajo la alternativa de crecimiento de ejes preferenciales de desarrollo. Los objetivos generales del PDU fueron: compactar y consolidar el área urbanizada; afianzar 
identidad de cada sector; proveer de equipamientos periféricos básicos (salud y educación); dignificar la población de villas; descentralizar funciones urbanas; integrar sectores inconexos; y revalorizar paisajística y patrimonialmente la ciudad (Irós, 1991). A su vez, se clasifican a las áreas de ciudad en tres categorías: área central, áreas intermedias y áreas periféricas (Figura 3). En cada una de ellas se proponen estrategias de intervención orientadas a corregir los problemas derivados del crecimiento histórico descontrolado y a potenciar las cualidades urbano-ambientales de cada sector. Para el área central se propone un plan de revalorización del patrimonio; en el área pericentral, se proponen obras de conectividad, infraestructura y espacios abiertos públicos; al área periférica se busca dotarla de servicios urbanos (centros educativos, deportivos y de salud, y espacios abiertos públicos) y vivienda (Figura 3). Con estas propuestas para el área pericentral y el área periférica, comienza a reforzarse desde la planificación cordobesa la idea de nuevas centralidades urbanas, complementarias al área central.

En el marco del PDU se sancionan cinco ordenanzas: Ocupación y preservación del Área Central; Fraccionamiento de tierras; Formas de ocupación del suelo (usos industriales y usos mixtos); Formas de ocupación del suelo (residencial); y Patrimonio Cultural. Estas cinco ordenanzas, que llevan más de tres décadas de vigencia, constituyen la base normativa bajo la cual se realizan diferentes modificaciones hasta la fecha. La primera modificación normativa importante que ocurre durante la gestión de Ramón B. Mestre es la sanción de la Ordenanza 8606/91 (Córdoba, 1991). Esta ordenanza incorpora la figura de Urbanizaciones Residenciales Especiales (URE), que se caracterizan por ser grandes urbanizaciones cerradas ubicadas en la periferia de la ciudad y por estar destinadas a un sector socioeconómico alto. A su vez, la ordenanza realiza la primera modificación importante a las zonificaciones propuestas en el marco normativo inicial. Esta modificación comienza a tensionar las ideas iniciales del Plan de Desarrollo Urbano por sus lógicas implícitas de entender la ciudad. Dentro de estas lógicas se referencian: la privatización del espacio urbano a gran escala (en particular del espacio abierto público, concesionado al agente urbanizador); la fragmentación físico-espacial mediante la incorporación de urbanizaciones cerradas; y la ampliación de la mancha urbana sobre la periferia con una ocupación en muy baja densidad.

En 1991, asume la gestión municipal Rubén Martí. Tras casi tres años de gestión su gestión incorpora
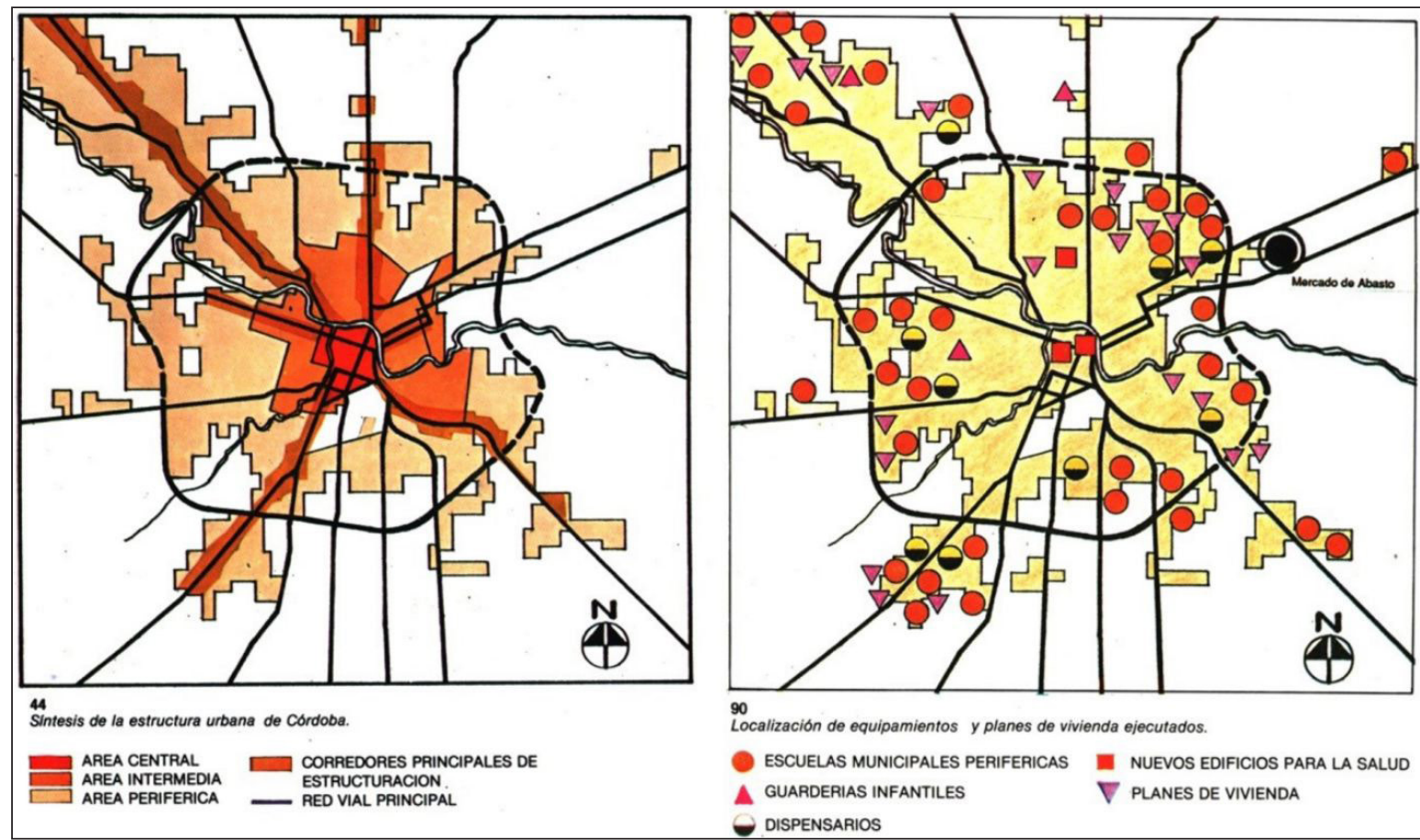

Figura 3 - Síntesis de la estructura urbana de Córdoba - Localización de equipamientos y planes de vivienda ejecutados Fuente: Elaboración propia a partir de gráficos de Desarrollo urbano: reflexiones y acciones, Irós (1991). 
el enfoque de la planificación estratégica. Esto representa una fecha temprana respecto al resto del contexto latinoamericano (Fernández Güell, 2007). Esta primera experiencia, desarrollada bajo el nombre de Plan Estratégico para la Ciudad (PEC) (Córdoba, 1996, 1999), representa cierto corrimiento de la preocupación de los problemas físicos-espaciales de la ciudad en función de una planificación avocada a la gestión y el diálogo entre los diferentes actores. Dicho corrimiento es apreciable principalmente en los objetivos del PEC, en los cuales toma especial importancia el desarrollo económico de la ciudad dentro del MERCOSUR (Figura 4) y el posicionamiento de la ciudad a partir de sus cualidades socio-ambientales, y no el ordenamiento de la ciudad como había sido el caso hasta entonces. No obstante, las preocupaciones físico-espaciales que se mencionan en el plan residen en retomar el desarrollo policéntrico de la ciudad que había comenzado en la gestión de Mestre con la dotación de servicios urbanos. A partir de una iniciativa por descentralizar la gestión municipal - tanto administrativa como físicamente -, surge la propuesta de crear los denominados Centros de Participación Comunal (CPC). Se pensó ubicar estratégicamente a estos edificios en la ciudad luego de dividir al municipio en nueve zonas de población similar (entre 100.000 y 200.000 habitantes) y se propuso un lenguaje arquitectónico de gran pregnancia y muy similar entre ellos (Figura 4). El objetivo de la implantación de estas nuevas sedes de la Municipalidad era, por un lado, disminuir la carga del área central y generar nuevas centralidades capaces de albergar el desarrollo económico la zona y, por el otro lado, llevar al Estado más cerca de los vecinos. En este sentido, los CPC funcionarían como espacios institucionales de convergencia y brindarían una oferta de servicios (municipales, provinciales y también privados), actividades (culturales, educativas, recreativas, etc.), programas sociales (de niñezy ancianidad) y también espacios físicos para reuniones de organizaciones barriales. En definitiva, la propuesta descentralizadora del PEC cambia ciertos aspectos de la propuesta de organización físico-espacial del EDOU y el PDU, en el sentido que pierden fuerza los ejes preferenciales de desarrollo y aparecen las centralidades de los CPC como nuevos polos de desarrollo.

\section{Segundo período: una alternancia de distintas fuerzas políticas e ideas}

El segundo período no se caracterizó por la continuidad de la política urbana sino por ser un período en que cada una de las tres gestiones, pertenecientes

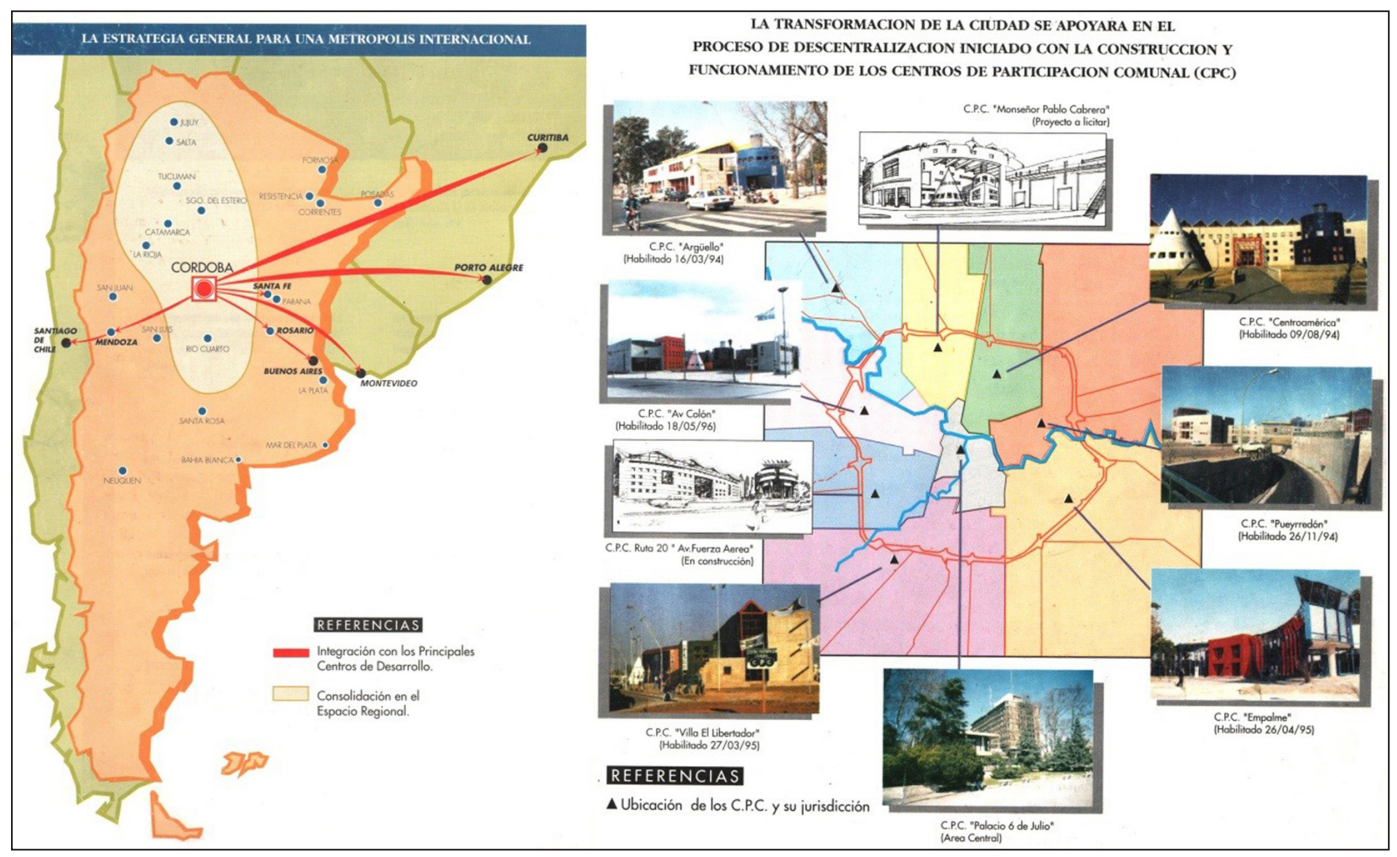

Figura 4 - La estrategia general para una metrópolis internacional - Localización y jurisdicción de los CPC Fuente: Elaboración propia a partir de gráficos de Plan estratégico para la Ciudad (PEC), Municipalidad de Córdoba (1996). 
a distintos partidos políticos, buscó transformar en mayor o en menor medida, con mayor o menor efectividad - los planes y marcos regulatorios.

En primer lugar, la gestión de Germán Kammerath (de Unión por Córdoba) ${ }^{3}$ propuso una breve pausa a la planificación estratégica, sin dejarla por completo de lado. Mediante la creación del Instituto de Planeamiento Urbano de Córdoba (IPUCOR) - que contó un equipo técnico de la Universidad Nacional de Córdoba con presencia de técnicos con formación específica en urbanismo -, se buscó constituir un ámbito de investigación y reflexión que tenía como objetivo elaborar un nuevo plan urbano ambiental ${ }^{4}$. En este sentido, se presentaron dos documentos: Diagnóstico y líneas estratégicas para el Plan Urbano Ambiental (Córdoba, 2001) y Formulación de lineamientos alternativos para el plan urbano ambiental (Córdoba, 2003), ambos con un claro énfasis físico-espacial.

Las líneas estratégicas resultantes del primer documento proponen: contener la expansión de la urbanización según el soporte natural y el desarrollo metropolitano; asegurar un funcionamiento bipolar del transporte; proteger las áreas productivas del cinturón verde; densificar la urbanización; revitalizar el centro histórico; resolver articuladamente el transporte metropolitano y accesibilidad vial; integrar social y funcionalmente la urbanización; y reformar la normativa vigente. De estas líneas, son las dos primeras las de mayor importancia en relación a cómo pensar la forma de crecimiento urbano. La primera línea - contener la expansión - denota que cambia la idea existente en otros planes de frenar a toda costa el crecimiento urbano por la idea de pensar qué sector de la periferia tendrá el soporte natural y las características metropolitanas para ser el nuevo espacio de crecimiento. La segunda línea -el funcionamiento bipolar- denota un modelo de crecimiento urbano a partir de dos polos fuera del centro histórico.

\footnotetext{
${ }^{3}$ Unión por Córdoba esuna alianza que, en su conformación inicial, incluyó al Partido Justicialista (PJ), de tendencia popular, liderado por José Manuel de la Sota; la Unión del Centro Democrático (UCeDé), de tendencia liberal, liderada por Germán Kammerath; el partido Acción para el Cambio (APEC), de tendencia desarrollista, liderado por Alfredo Keegan; y la Democracia Cristiana de Córdoba, liderada por Juan Brugge.

${ }^{4}$ Los profesionales mencionados en el documento son: Arq. Ma. Elena Foglia (dir.), Arq. Mario Donicelli, Arq. Fernando Díaz Terreno, Arq. Gabriela Pestrín.
}

Diagnóstico y líneas estratégicas orientativas para el Plan Urbano Ambiental (Córdoba, 2001) parte de proponer uno de estos dos polos en el Noroeste de la ciudad, en la zona conocida como Chateau Carreras; el motivo residía en la oportunidad de localizar allí la nueva sede del gobierno provincial (Figura 5) ${ }^{5}$.

En el segundo documento se plasma un análisis preliminar de ventajas y desventajas de siete sectores de la periferia del municipio para poder determinar cuáles podrían ser ejes preferenciales de expansión futura. De este análisis se descartan tres ejes por no tener las características ambientales y funcionales. Luego se modelan alternativas de configuración y de proyectos emergentes necesarios, a modo de poder establecer un análisis comparativo del modelo de ciudad resultante una vez seleccionada la alternativa de anexión de sectores más conveniente para transformarse en el nuevo eje preferencial de expansión.

De modo general, se observa que en las acciones propuestas por el IPUCOR se renueva la idea de pensar la ciudad a partir de ejes preferenciales de desarrollo de la planificación de los años '70, llamándolos en esta ocasión "de expansión", entendiendo que estos nuevos anexos en la mancha urbana serán los responsables de alojar la mayor parte de un crecimiento habitacional proyectado en medio millón de personas en 20 años ${ }^{6}$.

En segundo lugar, la gestión de Luis Juez (Partido Nuevo), cierra el IPUCOR para desarrollar una segunda instancia de planificación estratégica mediante el Plan Estratégico para la ciudad de Córdoba. Una ciudad como su gente (PECba) (Córdoba, 2005). El PECba no introduce una nueva forma de pensar el espacio urbano de la ciudad ni plantea un nuevo modelo físico-espacial para la ciudad, es un documento de carácter analítico. Sin embargo, propone ciertas transformaciones a ideas desarrolladas en sus planes antecesores. Complementariamente, el PECba

\footnotetext{
${ }^{5}$ El proyecto de la nueva sede del gobierno provincial en la zona del Chateau Carreras no se llevó a cabo. En su lugar, el nuevo Centro Cívico fue construido en los límites del Centro Histórico, sobre predios ferroviarios colindantes a la estación de trenes Mitre.

${ }^{6}$ Según datos del Censo Nacional 2001 y 2010 (INDEC, 2001, 2010), el aumento poblacional en el Municipio de Córdoba entre estas fechas fue de 45.022 personas. Es decir que el municipio, para el que estaba proyectado un crecimiento aproximado de 250.000 personas por década, creció tan solo la quinta parte de esa proyección durante la primera de esas décadas.
} 

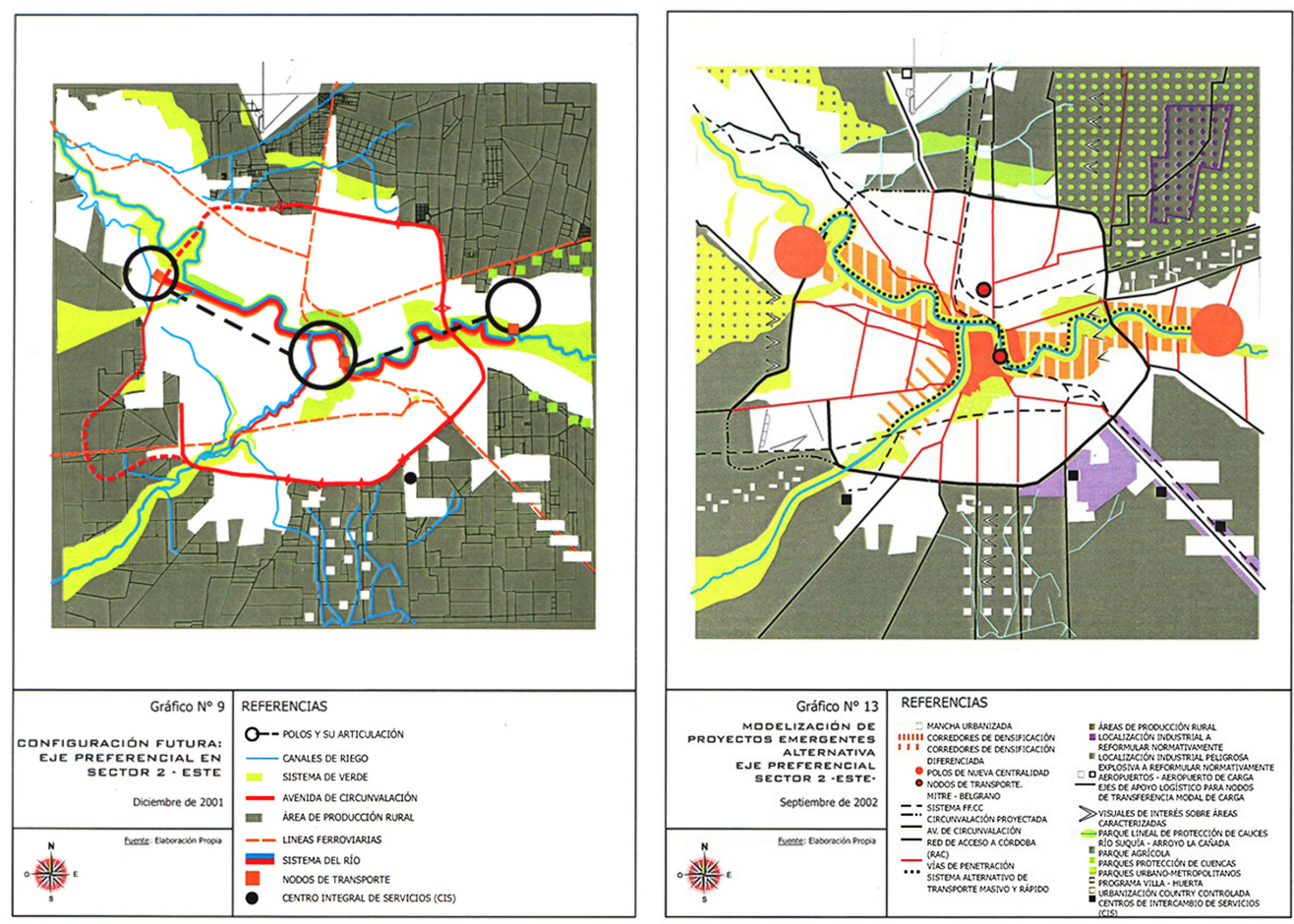

Figura 5 - Configuración futura: eje preferencial sector 2 (suroeste) - Modelación de proyectos emergentes. Alternativa eje preferencial sector 2 (suroeste) Fuente: Elaboración propia a partir de gráficos de Formulación de lineamientos alternativos para el plan urbano ambiental, Municipalidad de Córdoba (2003).

desarrolla una serie de cuatro Planes integrales de desarrollo zonal y un proyecto urbano denominado Portal del Abasto, que propone la revitalización del norte del área central hasta cruzar el Río Suquía (Figura 6) ${ }^{7}$. También aparecen con gran importancia los proyectos complementarios propuestos por el sector privado; entre ellos, se destacan los grandes centros comerciales y hoteles internacionales de lujo. No obstante, el documento no menciona cuál es el

\footnotetext{
${ }^{7}$ Para 2005, fecha en la que fue publicado el documento, los Planes integrales de desarrollo zonal que estaban puestos en marcha eran cuatro: Revalorización del pasaje Aguaducho/ Paseo de la Reforma Universitaria; Programa Zonal de Argüello; Recuperación y puesta en valor del Área Central de la Ciudad; y Programa de revitalización integral de Alta Córdoba y barrios aledaños. Los primeros dos tienen una escala pequeña de intervención y el documento no deja en claro qué se propone en ellos. Para el Área Central, se proponen acciones como la incorporación de seguridad, iluminación y mejoramiento de sus calles relevantes en la escala municipal. En este sentido, es un proyecto que no propone cambios físico-espaciales. Por su parte, el programa de Alta Córdoba propone la incorporación de densidades edilicias medias, un polo de atracción de actividades culturales y un Proyecto urbano de los Predios Ferroviarios.
}

grado de articulación entre estos planes, proyectos urbanos y proyectos arquitectónicos.

El PECba recupera los principios claves de su antecesor, el PEC. En primer lugar - como ejemplos claros de planificación estratégica dentro del contexto latinoamericano en estas décadas - ambos piensan a la ciudad desde su posicionamiento económico dentro de un marco fuertemente sesgado por la globalización. Mientras que el PEC propone que el nuevo rol del Estado será la generación de condiciones para el desarrollo económico local mediante inversiones extranjeras, el PECba define que la ciudad "impulsará el corredor bioceánico del Mercosur hacia el mundo" (Córdoba, 2005, p. 83). En segundo lugar, las "líneas estratégicas" propuestas por el PECba - La gente, La producción y el trabajo, La dinámica urbana y El capital natural - son equivalentes a los "ejes" que propone el PEC - Social, Económico, Urbano y Ambiental -; consecuentemente, el sesgo de los proyectos que componen ambos planes es bien similar.

En consonancia con estudios y propuestas de 2001 y 2003 realizados por el IPUCOR, que refuerzan la alerta sobre el crecimiento desmedido de la ciudad, el PECba define una estrategia territorial que está orientada al generar un desarrollo más compacto de 


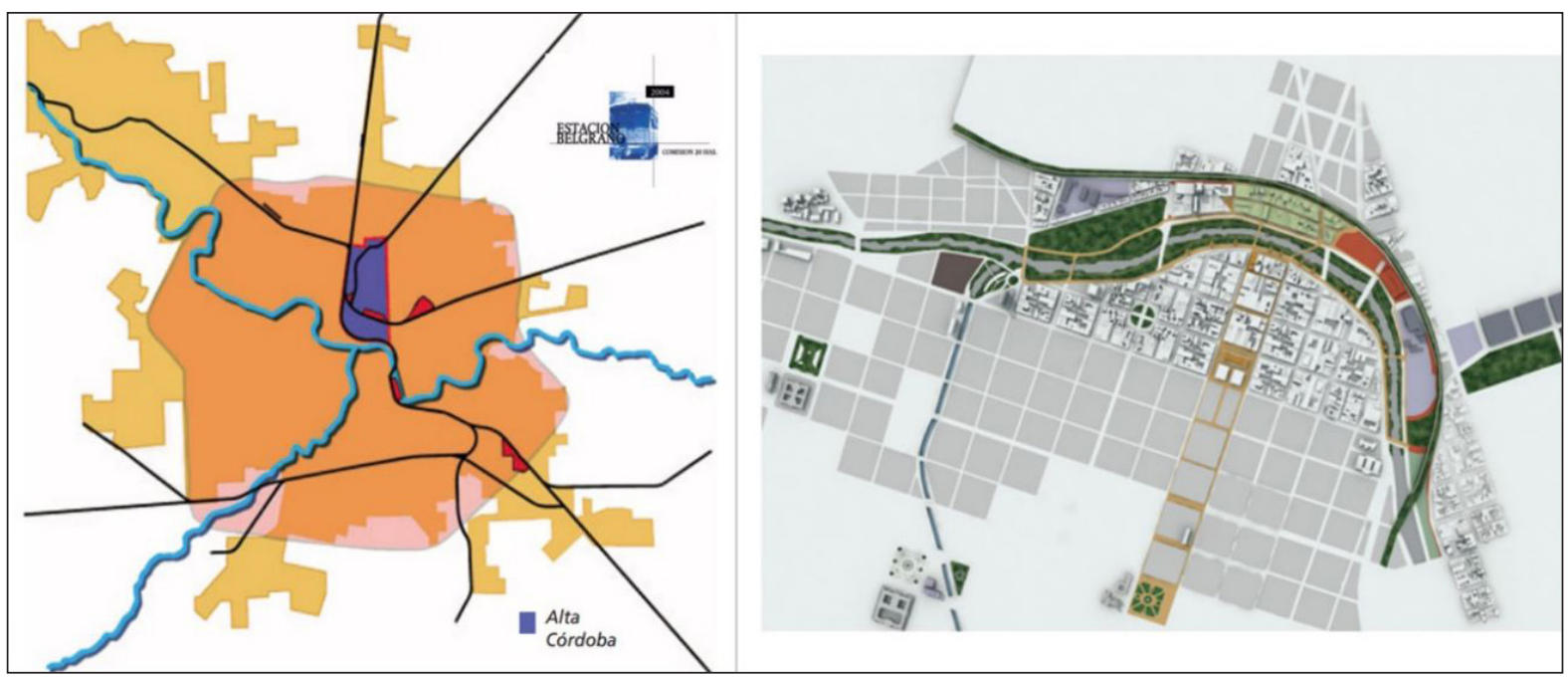

Figura 6 - Esquema del Área de intervención en Bº Alta Córdoba - Planimetría del Portal del Abasto Fuente: Elaboración propia a partir de gráficos de Plan Estratégico de la ciudad de Córdoba. Una ciudad como su gente (PECba), Municipalidad de Córdoba (2005).

la ciudad, frenando su crecimiento por expansión a baja densidad (Córdoba, 2005, p. 19).

Existe también un punto de contacto entre el PECba y el PDU de la gestión de Ramón B. Mestre que es el interés explícito de ambos planes por el pragmatismo. Mientras que "[...] el PECba no es un ejercicio teórico, es un proceso que invita y requiere pasar a la acción [...]" (Córdoba, 2005, p. 9) en el PDU se reconoce el rechazo hacia una actitud

[...] que dedica mucho tiempo y energía a la elaboración de un completo y preciso diagnóstico, pero en desmedro de la acción directa sobre los problemas de la ciudad [...] (Irós, 1991, p. 59).

En tercer lugar, en diciembre de 2007, asume la gestión municipal de Daniel Giacomino (Frente Cívico y Social) ${ }^{8}$. Un año más tarde, la Municipalidad de Córdoba -en conjunto con un equipo técnico de la Universidad Nacional de Córdoba - presenta las Bases para el Plan Director de la Ciudad de Córdoba (Córdoba 2020)

\footnotetext{
${ }^{8}$ El Frente Cívico y Social (FCyS) fue una alianza que, en su conformación inicial, incluyó al Partido Nuevo (liderado por Luis Juez), al Partido Comunista, al Partido Intransigente, al Partido de la Victoria, entre otros. Giacomino, quien fue electo intendente dentro de este frente, buscó la continuidad de políticas del FCyS. Debido a conflictos políticos en el ámbito provincial y nacional, Juez y su espacio político se distanciaron de Giacomino. Este último se quedó prácticamente sin concejales municipales propios para gobernar y fue progresivamente alineándose dentro del esquema nacional del Frente para la Victoria (que en ese entonces presidía el gobierno nacional).
}

(Córdoba, 2008) que postulan la necesidad de revisión de la situación de la planificación y la regulación en Córdoba, con el objetivo de elaborar un nuevo plan urbano. Estas bases no se transformaron efectivamente en un plan urbano aprobado por ordenanza, como sí había sucedido con el EDOU en 1978.

El documento deja en claro que se precisa un Estado Municipal que promueva y genere propuestas de desarrollo urbano, y no simplemente regule y controle. Para ello, se formula una estrategia general de ordenación del territorio atenta a la dinámica metropolitana y se desprenden lineamientos de acción. Estas bases alientan una intervención sobre las normativas existentes para lograr una densificación

[...] sobre la base de la conectividad existente o futura con la finalidad de lograr valores de concentración de población que hagan sustentable las provisiones de servicios, equipamientos y los sistemas de transporte público (Córdoba, 2008, p. 11).

El documento desarrolla proyectos de ordenación e incorporación de usos mixtos para nuevas áreas de intervención estratégica identificadas en 23 polígonos y 16 nodos. Los polígonos de propuestos recuperan la noción de áreas estratégicas para la intervención de las Áreas Especiales de la normativa vigente. Sin embargo, estas áreas son entendidas de manera más abarcativa en términos de la superficie planificada, su alcance y el tipo de renovación que proponen. Más aún, distinto 

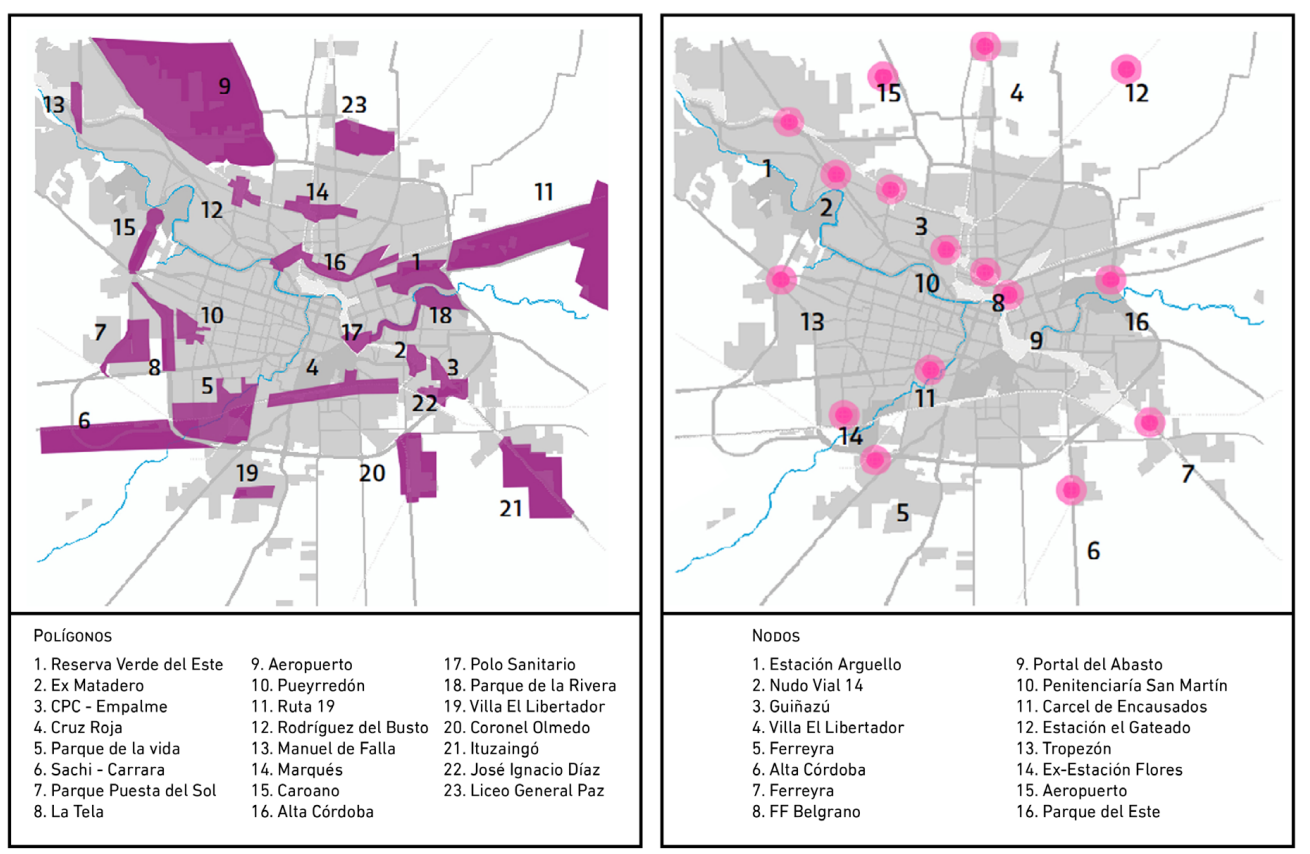

Figura 7 - Nuevos espacios de intervención estratégica: Polígonos - Nuevos espacios de intervención estratégica: Nodos Fuente: Elaboración propia a partir de gráficos de Bases para el Plan Director. Lineamientos y estrategia general para el reordenamiento del territorio, Municipalidad de Córdoba, \& Universidad Nacional de Córdoba (2008).

de las Áreas Especiales, Córdoba 2020 cuenta con lineamientos proyectuales para ellas.

A diferencia de la propuesta del PDU de clasificar la ciudad en tres categorías (área central, áreas intermedias y áreas periféricas), estas bases incorporan una nueva categoría: las áreas pericentrales ${ }^{9}$. Para cada una de estas cuatro áreas se plantean diferentes planes sectoriales para sus barrios. Por ejemplo, para los barrios periféricos se busca la integración funcional-espacial de urbanizaciones insulares mediante la habilitación de expansiones acotadas en áreas específicas, y la generación y consolidación de centros barriales y espacios públicos. Para los barrios pericentrales tradicionales se busca orientar los procesos de renovación dirigidos por el mercado inmobiliario para lograr una consolidación barrial con mayor densidad habitacional.

Un aporte de esta instancia de planificación es haber incorporado a la movilidad como una dimensión importante a tener en cuenta. En efecto, siguiendo con el objetivo de generar un Estado Municipal presente,

\footnotetext{
${ }^{9}$ En Córdoba 2020 las aéreas pericentrales serán lo que para el PDU eran las intermedias, y las anteriormente periféricas se dividirán ahora entre intermedias y periféricas.
}

se revisa el sistema de transporte urbano público de colectivos y se proponen dos nuevas modalidades: un sistema de ferro urbano sobre antiguas vías de tren y un subterráneo bajo la Av. Colón, un importante eje de desarrollo Este-Oeste.

En general, Córdoba 2020 marca objetivos generales claros y pasa directo a una serie de lineamientos concretos bajados a recortes espaciales puntuales de la ciudad (como los nodos o los polígonos representados en la Figura 7) o desarrollados en torno a una problemática particular (como el servicio de transporte o la infraestructura). Como ya se mencionó, estos lineamientos son los que a futuro deberían haberse tenido en cuenta para la formulación de un nuevo plan urbano.

El último documento de planificación municipal de esta gestión es el Programa de densificación urbana (Córdoba, 2010). Este documento, que tiene un carácter preliminar, propone la necesidad de incrementar la densidad habitacional de la ciudad para intentar combatir los elevados costos de extensión de infraestructura y servicios, y de ocupación de áreas productivas, problemas derivados de la extensión de la mancha urbana a baja densidad. El foco está puesto en la realización de ciertas modificaciones normativas 
leves que posibiliten, por un lado, intensificar el uso residencial y, por el otro, introducir nuevas tipologías de vivienda colectiva. Se propone un tipo de intervención en corredores y áreas. Para los corredores se respeta el esquema existente, incorporando algunos nuevos y de la extensión de otros (Figura 8). A su vez, se plantea que la unidad de diseño de los corredores sea toda la manzana y no solamente los lotes frentistas del mismo. Para las áreas las modificaciones normativas incluyen la búsqueda por liberar suelo en planta baja, mayor cantidad de unidades por lote, mayor factor de ocupación del suelo y se propone permitir la vivienda colectiva en las Urbanizaciones Residenciales Especiales (Figura 8). En general, el programa no plantea modificaciones estructurales al esquema de ordenamiento ni a la manera de entender la ciudad, sino que propicia un uso más eficiente del suelo - en términos cuantitativos - mediante pequeños cambios normativos.

\section{Otros documentos de planificación para Córdoba}

Cabe destacar que desde la Presidencia de la Nación se publicaron otros documentos de planificación de escala nacional: el Plan Estratégico Territorial - Avance I (Argentina, 2008), el Plan Estratégico Territorial Edición Bicentenario (Argentina, 2010) y el Plan Estratégico Territorial - Avance II (Argentina, 2011). Estas tres instancias de planificación - que muestran fuerte sesgo de desarrollo económico local y regional, desde una mirada consiente de la dimensión físico-espacial - incorporan una escala que excede al municipio de Córdoba, pero donde el mismo juega un papel fundamental en la integración del territorio a escala nacional.

Finalmente, es importante también mencionar la publicación del documento Lineamientos del Plan Estratégico Urbano Territorial de la Región Metropolitana de Córdoba (Córdoba, 2012), elaborado por el Instituto de Planificación del Área Metropolitana de la Provincia de Córdoba (IPLAM). Si bien el documento del plan fue publicado a principios de 2012 (es decir, técnicamente fuera del segundo período que se analiza en este artículo) y, a su vez, es de escala metropolitana, su formulación y dos leyes provinciales asociadas a estos lineamientos que se aprueban durante ese proceso de planificación metropolitana sí pertenecen al período en cuestión. Este documento -a pesar de que puede recibir fuertes críticas respecto a su marco teórico, a las propuestas que genera, a su desconocimiento de instancias de planificación metropolitanas previas y a
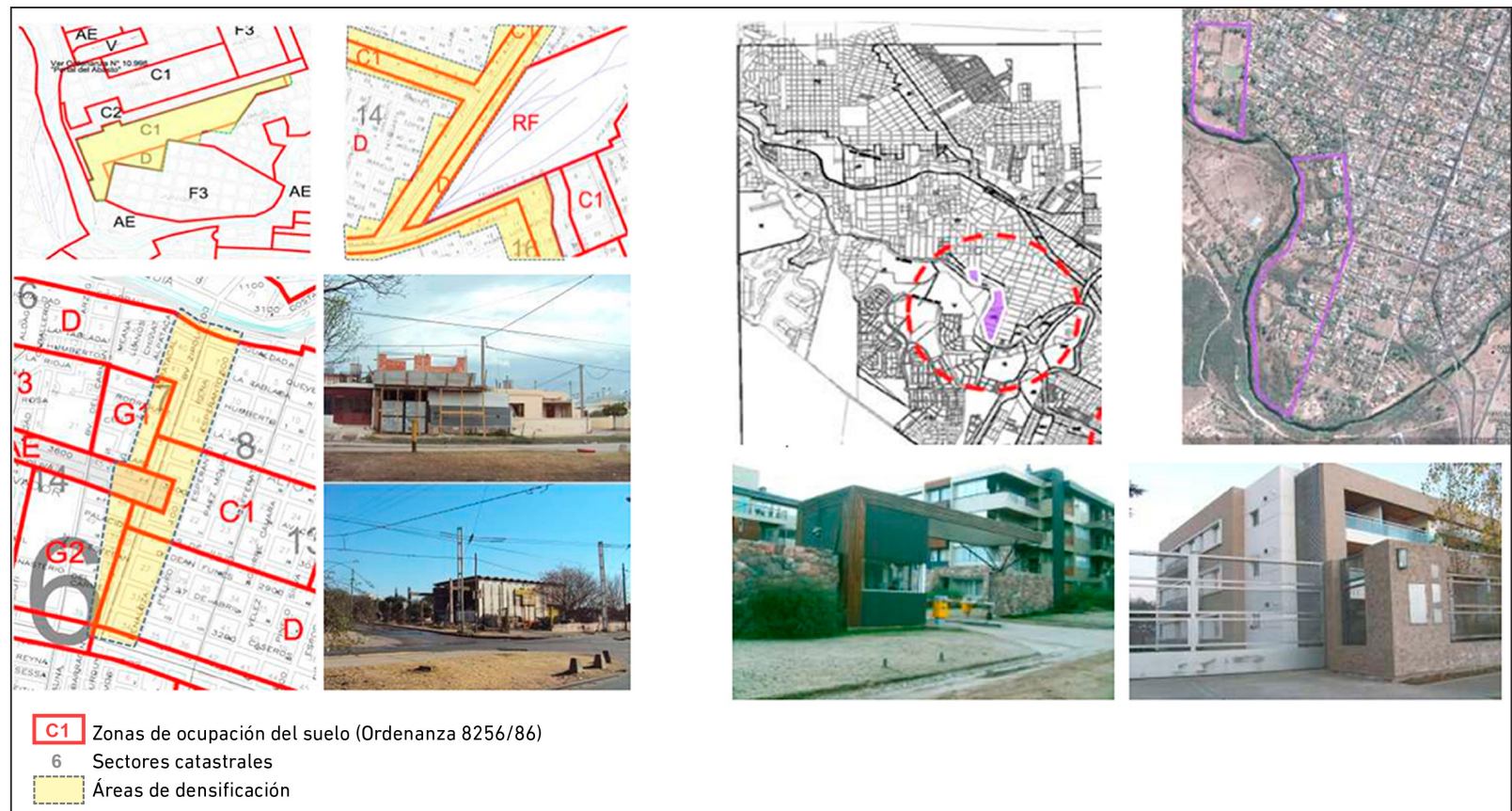

Figura 8 - Modificaciones normativas sobre corredores - Modificaciones normativas sobre áreas Fuente: Elaboración propia a partir de gráficos de Programa de densificación urbana, Municipalidad de Córdoba (2010). 
ciertas lecturas físico-espaciales que realiza sobre el área metropolitana de Córdoba - resalta por recuperar a la escala metropolitana e intermunicipal como escala específica de planificación y porque, a partir de él, se sancionaron dos leyes particulares provinciales con influencia directa en el Municipio de Córdoba: una de movilidad y otras de uso de suelo.

\section{Situación emergente: frente a la falta de consenso en la planificación urbana}

¿A dónde nos sitúa el curso de la evolución que se ha analizado? Quizás la principal conclusión a la que se arriba luego de realizar estas lecturas es que Córdoba carece de una única manera de pensar el espacio urbano de la ciudad y que existen escasos consensos entre las diferentes gestiones municipales sobre cuál debería ser el modelo físico espacial a seguir. En los documentos de planificación urbana analizados se destaca la repetitiva preocupación por frenar el crecimiento de la ciudad por expansión que, en su mayoría, es baja densidad habitacional. La idea más persistente desde el retorno a la democracia (y, tal vez, una de las pocas ideas con continuidad) en respuesta a esta problemática es la de los ejes preferenciales de desarrollo. Este modelo de crecimiento, se repite y transforma a lo largo de la mayoría de los documentos de planificación de las diferentes gestiones municipales con mayor o menor presencia, pero con distintos significados. A su vez, los ejes están plasmados desde las normativas de base que, con sus modificaciones, regulan el espacio urbano de la capital cordobesa desde 1985.

En este sentido, los cambios en las fuerzas políticas que gestionaron la municipalidad entre 1983 y 2011, y sus respectivos cambios en la manera de pensar la ciudad no han colaborado en llegar al consenso. Si bien en el primer período analizado existe una notable continuidad de políticas urbanas, a partir del segundo período, cada gestión ha buscado diferenciarse de la anterior. En cierta forma cada gestión ha comenzado su proceso de planificación prácticamente desde cero, a veces hasta ignorando por completo lo realizado por la gestión inmediatamente anterior. Como se resaltó a lo largo del artículo, sí es posible encontrar ideas aisladas y abordajes similares que se repiten. Sin embargo, muy pocas veces se reconocen las fuentes de las ideas y éstas no son siempre aplicadas de manera coherente con las nuevas propuestas.

\section{Referências}

Argentina. Presidencia de la Nación. (2008). Plan Estratégico Territorioal. Avance I. Buenos Aires: Presidencia de la Nación.

Argentina. Presidencia de la Nación. (2010). Plan Estratégico Territorial Bicentenario. Buenos Aires: Presidencia de la Nación.

Argentina. Presidencia de la Nación. (2011). Plan Estratégico Territorial Avance II. Buenos Aires: Presidencia de la Nación.

Caporossi, C. (2008). Planes y Normativa de las ciudades Planificación y crecimiento urbano en la ciudad de Córdoba. Café de Las Ciudades, (73), 1-12. Recuperado el 14 de enero de 2014, de www.cafedelasciudades.com.ar/ planes_normativas_73_p.htm

Córdoba. Municipalidad de la Ciudad. (1973). Diagnóstico tentativo y alternativas de desarrollo físico para la ciudad de Córdoba. Córdoba: Municipalidad de la Ciudad.

Córdoba. Municipalidad de la Ciudad. (1978). Esquema de ordenamiento urbano para la ciudad de Córdoba: año 2000 (EDOU). Córdoba: Municipalidad de la Ciudad.

Córdoba. Municipalidad de la Ciudad. (1979). Esquema de ordenamiento metropolitano (EDOM). Córdoba: Municipalidad de la Ciudad.

Córdoba. Municipalidad de la Ciudad. (1991). Ordenanza $n^{\circ}$ 8606/91 - Urbanizacion Residencial Especial. Córdoba: Municipalidad de la Ciudad.

Córdoba. Municipalidad de la Ciudad. (1996). Plan estratégico para la Ciudad (PEC). Córdoba: Municipalidad de la Ciudad.

Córdoba. Municipalidad de la Ciudad. (1999). Plan estratégico para la Ciudad (PEC). Córdoba: Municipalidad de la Ciudad.

Córdoba. Municipalidad de la Ciudad. (2001). Diagnóstico y líneas estratégicas orientativas para el Plan Urbano Ambiental. Córdoba: Municipalidad de la Ciudad.

Córdoba. Municipalidad de la Ciudad. (2003). Formulación de lineamientos alternativos para el plan urbano ambiental. Córdoba: Municipalidad de la Ciudad.

Córdoba. Municipalidad de la Ciudad. (2005). Plan Estratégico de la ciudad de Córdoba. Una ciudad como su gente (PECba). Córdoba: Municipalidad de la Ciudad.

Córdoba. Municipalidad de la Ciudad, \& Universidad Nacional de Córdoba. (2008). Bases para el Plan Director 
Lineamientos y estrategia general para el reordenamiento del territorio (Córdoba 2020). Córdoba: Municipalidad de la Ciudad.

Córdoba. Municipalidad de la Ciudad. (2010). Programa de densificación urbana. Córdoba: Municipalidad de la Ciudad.

Córdoba. Gobierno de la Provincia. (2012). Lineamientos del plan estratégico urbano territorial de la región metropolitana de Córdoba. Córdoba.

Díaz Terreno, F. (2011). Los territorios periurbanos de Córdoba. Entre lo genérico y lo específico. Revista Iberoamericana de Urbanismo, 5, 65-84.

Fernández Güell, J. M. (2007). 25 años de planificación estratégica de ciudades. Ciudad y Territorio. Estudios Territoriales, 39(154), 621-637.
Instituto Nacional de Estadísticas y Censos - INDEC. (2001). Censo Nacional de Población, Hogares y Viviendas. Buenos Aires: Instituto Nacional de Estadísticas y Censos.

Instituto Nacional de Estadísticas y Censos - INDEC. (2010). Censo Nacional de Población, Hogares y Viviendas. Buenos Aires: Instituto Nacional de Estadísticas y Censos.

Irós, G. (1991). Desarrollo urbano: reflexiones y acciones. Córdoba: Editorial Municipal de la Ciudad de Córdoba.

Venturini, E. J., Ávila, V. D., \& Terreno, C. (2010). Transformaciones urbano-territoriales y sustentabilidad del desarrollo en la ciudad de Córdoba 1910-2010. Arquisur Revista, 5(7), 74-93.

Recibido: Jun. 30, 2016

Aprobado: Sept. 28, 2016 